\title{
Editorial: Resource Allocation in Cloud-Radio Access Networks and Fog-Radio Access Networks for B5G Systems
}

\begin{abstract}
Hayssam Dahrouj ${ }^{1 *}$, Ahmed Douik ${ }^{2}$, Mohammed El-Hajjar ${ }^{3}$, Megumi Kaneko ${ }^{4}$, Yonghui Li ${ }^{5}$, Duy T. Ngo ${ }^{6}$, Mohamed Saad ${ }^{7}$, Kaiming Shen ${ }^{8}$ and Jun Zhang ${ }^{9}$

${ }^{1}$ King Abdullah University of Science and Technology, Thuwal, Saudi Arabia, ${ }^{2}$ California Institute of Technology, Pasadena, CA, United States, ${ }^{3}$ University of Southampton, Southampton, United Kingdom, ${ }^{4}$ National Institute of Informatics, Chiyoda-ku, Japan, ${ }^{5}$ The University of Sydney, Darlington, NSW, Australia, ${ }^{6}$ The University of Newcastle, Callaghan, NSW, Australia, ${ }^{7}$ University of Sharjah, Sharjah, United Arab Emirates, ${ }^{8}$ The Chinese University of Hong Kong, Shenzhen, China, ${ }^{9}$ Hong Kong University of Science and Technology, Hong Kong, China
\end{abstract}

Keywords: Fog-radio access network (F-RAN), cloud-radio access network (C-RAN), resource allocation, beyond $5 G$ (B5G) networks, $6 G$ networks

\section{Editorial on the Research Topic}

Resource Allocation in Cloud-Radio Access Networks and Fog-Radio Access Networks for B5G Systems

\section{OPEN ACCESS}

Edited and reviewed by: Hong-Chuan Yang,

University of Victoria, Canada

*Correspondence:

Hayssam Dahrouj

hayssam.dahrouj@gmail.com

Specialty section:

This article was submitted to

Wireless Communications,

a section of the journal

Frontiers in Communications and

Networks

Received: 11 October 2021 Accepted: 01 November 2021 Published: 19 November 2021

Citation:

Dahrouj H, Douik A, El-Hajjar M, Kaneko M, Li Y, Ngo DT, Saad M,

Shen K and Zhang J (2021) Editorial:

Resource Allocation in Cloud-Radio

Access Networks and Fog-Radio

Access Networks for B5G Systems.

Front. Comms. Net 2:793084.

doi: 10.3389/frcmn.2021.793084
The ubiquitous demand for high data rate services necessitates breakthroughs in network system architecture. With a progressive move towards full spectrum reuse and a positive trend in smallcell deployment, cloud-radio access networks (CRANs) and fog-radio access networks (FRANs) become essential in large-scale interference management for beyond 5G (B5G) wireless systems. By connecting the base-stations (BSs) to the centralized cloud, CRANs provide a strong ability to allocate resources in a coordinated way across base-stations through inter-base-station coordination and through the proper use of the interconnecting fronthaul links. CRANs combine the ability of joint signal processing of users' data, and the strong capability of computing high complexity algorithms that standalone base-stations are incapable of. FRANs, on the other hand, are designed to reduce the delay caused by the fronthaul links by pushing control and storage abilities to the network edge. Such functionality of FRANs allows mitigating CRANs' latency and signaling overhead issues by moving some of the network intelligence closer to the network edge.

Such interplay between CRANs' strong centralized processing and FRANs' distributed intelligence capabilities provides a fertile arena for efficient resource allocation strategies. This is particularly the case since both CRANs and FRANs (albeit at different scales) make optimized resource sharing strategies feasible between the different entities through the cloud, by means of jointly encoding/decoding the messages using downlink/uplink coordinated resource allocation techniques. Recent studies have also suggested that CRANs and FRANs platforms are particularly suitable for the practical implementation of rate-splitting strategies, i.e., by dividing each user message into two parts, a private part decodable at the intended user only, and a common part which can be decoded by another user, for the purpose of reducing large-scale interference.

Our research topic (RT) is, therefore, devoted to investigating large scale interference management techniques in the context of CRANs and FRANs for B5G systems, through the proper optimization of resource allocation and rate-splitting techniques, both jointly and separately. 
The rest of this editorial sheds light on each of the four highquality manuscripts that feature at our RT.

We start with the paper co-authored by Li et al., entitled "Analog Radio Over Fiber Aided C-RAN: Optical Aided Beamforming for Multi-User Adaptive MIMO Design." In this paper, the central unit and the remote radio heads are connected by optical fiber links. Additionally, multiple-input-multipleoutput (MIMO) techniques are prevalent for the sake of improving the system's performance, where beamformingbased MIMO is used for boosting the signal-to-noise ratio (SNR) and alleviating the interference. However, the large number of analogue phase-shifters required for the beamforming design suffers from reduced phase resolutions and degraded noise figures, in addition to the fact that phaseshifter based beamforming suffers from the beam-squinting phenomenon. Therefore, this article proposes an analogue radio over fiber (A-RoF) assisted technique for supporting future low-cost, yet high-performance CRAN design. The article further employs the photonics aided beamforming technique to support a reconfigurable MIMO system in the A-RoF based CRAN architecture, under different spatial multiplexing scenarios.

A second paper, co-authored by Ahmed et al., entitled "Power Minimization Using Rate Splitting With Statistical CSI in CloudRadio Access Networks," focuses on the design aspect of CRANs by investigating optimal resource allocation towards minimizing the transmit power, while ensuring a minimum quality of service (QoS). This paper assumes that a central processor (CP) is connected to the BSs via limited capacity fronthaul links, and that the $\mathrm{CP}$ has only statistical knowledge of channel state information (CSI). The paper proposes a multiple access scheme, in which the $\mathrm{CP}$ applies a rate-splitting strategy at the cloud level. The considered problem is stochastic, complex, and non-convex. This paper addresses the problem intricacies through an iterative approach that leverages both the sample average approximation (SAA) technique and the weightedminimum mean squares error (WMMSE) algorithm to obtain a stationary point of the optimization problem at the asymptotic regime.

Along the same direction, Reifert et al. consider a rate-splitting multiple access (RSMA) scheme in the paper entitled "RateSplitting Multiple Access in Cache-Aided Cloud-Radio Access Networks." The paper proposes an RSMA-based multigroup multicast transmission scheme tailored to a cache-enabled cloud-radio access network. Here, RSMA is used as an efficient interference management strategy, and local caches are employed at the BSs, in order to store popular contents close to the users. The network users are grouped based on their content requests, and each group is served by a set of BSs controlled by a CP, which only has access to the statistical CSI. To guarantee multicast group fairness, the authors focus on maximizing the minimum rate among all groups, by jointly optimizing clustering and precoding. A mixed-integer nonlinear stochastic program (MINLSP) is subsequently solved by a heuristic clustering algorithm, as well as an iterative algorithm combining SAA and WMMSE for precoder design, thereby unveiling the potential of RSMA in cache-aided CRANs.

Last but not least, Mershad et al. address the prospects of highaltitude platform systems (HAPS) in "Cloud-Enabled HighAltitude Platform Systems: Challenges and Opportunities." HAPS, deployed within the stratosphere layer, are expected to better serve ground networks due to their large footprint and line of sight connection. By further hosting and offering cloud applications from within the HAPS, cloud providers can reach a larger number of users in urban areas, and enable cloud users that reside in rural and disconnected areas to benefit from the cloud computing capabilities. In this paper, the authors discuss the cloud applications that utilize the HAPS-cloud platform to extend their reach and enhance their services. This paper further describes how specific types of cloud applications can be deployed within the HAPS cloud to enhance their capabilities and improve their quality of service. The article discusses the challenges of such deployment, and provides several directions for future research on this topic, which promises to be a prolific area in the context of B5G/6G systems architecture.

\section{AUTHOR CONTRIBUTIONS}

All authors listed have made a substantial, direct, and intellectual contribution to the work and approved it for publication.

Conflict of Interest: The authors declare that the research was conducted in the absence of any commercial or financial relationships that could be construed as a potential conflict of interest.

Publisher's Note: All claims expressed in this article are solely those of the authors and do not necessarily represent those of their affiliated organizations, or those of the publisher, the editors and the reviewers. Any product that may be evaluated in this article, or claim that may be made by its manufacturer, is not guaranteed or endorsed by the publisher.

Copyright (c) 2021 Dahrouj, Douik, El-Hajjar, Kaneko, Li, Ngo, Saad, Shen and Zhang. This is an open-access article distributed under the terms of the Creative Commons Attribution License (CC BY). The use, distribution or reproduction in other forums is permitted, provided the original author(s) and the copyright owner(s) are credited and that the original publication in this journal is cited, in accordance with accepted academic practice. No use, distribution or reproduction is permitted which does not comply with these terms. 